\title{
Evaluation of function predictions by PFP, ESG, and PSI-BLAST for moonlighting proteins
}

\author{
Ishita K Khan¹, Meghana Chitale ${ }^{1}$, Catherine Rayon ${ }^{3}$, Daisuke Kihara ${ }^{2,1^{*}}$ \\ From Great Lakes Bioinformatics Conference 2012 \\ Ann Arbor, MI, USA. 15-17 May 2012
}

\begin{abstract}
Background: Advancements in function prediction algorithms are enabling large scale computational annotation for newly sequenced genomes. With the increase in the number of functionally well characterized proteins it has been observed that there are many proteins involved in more than one function. These proteins characterized as moonlighting proteins show varied functional behavior depending on the cell type, localization in the cell, oligomerization, multiple binding sites, etc. The functional diversity shown by moonlighting proteins may have significant impact on the traditional sequence based function prediction methods. Here we investigate how well diverse functions of moonlighting proteins can be predicted by some existing function prediction methods.

Results: We have analyzed the performances of three major sequence based function prediction methods, PSI-BLAST, the Protein Function Prediction (PFP), and the Extended Similarity Group (ESG) on predicting diverse functions of moonlighting proteins. In predicting discrete functions of a set of 19 experimentally identified moonlighting proteins, PFP showed overall highest recall among the three methods. Although ESG showed the highest precision, its recall was lower than PSI-BLAST. Recall by PSI-BLAST greatly improved when BLOSUM45 was used instead of BLOSUM62.
\end{abstract}

Conclusion: We have analyzed the performances of PFP, ESG, and PSI-BLAST in predicting the functional diversity of moonlighting proteins. PFP shows overall better performance in predicting diverse moonlighting functions as compared with PSI-BLAST and ESG. Recall by PSI-BLAST greatly improved when BLOSUM45 was used. This analysis indicates that considering weakly similar sequences in prediction enhances the performance of sequence based AFP methods in predicting functional diversity of moonlighting proteins. The current study will also motivate development of novel computational frameworks for automatic identification of such proteins.

\section{Background}

The ever growing genome sequencing data and the overwhelming development of genome sequencing technologies have boosted the development of computational techniques and resources for protein function prediction $[1,2]$. The traditional sequence based functional annotation is based on the concept of homology $[3,4]$ or motif/ domain searches [5-7]. Some recent Automatic Function Prediction (AFP) methods such as PFP [8,9], ESG [10], Gotcha [11], GOFigure [12], and ConFunc [13] use the

\footnotetext{
* Correspondence: dkihara@purdue.edu

2Department of Biological Sciences, Purdue University, 915 W. State Street, West Lafayette, Indiana 47907, USA

Full list of author information is available at the end of the article
}

Gene Ontology (GO) hierarchy. On the other hand, SIFTER [14], FlowerPower [15] and Orthostrapper [16] employ phylogenetic trees to transfer functions to target genes in the evolutionary context. There are other function prediction methods that consider co-expression patterns [17-21], 3D structures of proteins [22-30] as well as protein-protein interaction networks [31-36].

Although existing AFP methods show numerous successful predictions, moonlighting proteins may pose a challenge as they are known to show more than one function that are diverse in nature [37-39]. The varied functional behavior of these proteins can be due to localization within the cell, expression by different cell types, binding of a cofactor, oligomerization, complex formation, or
() Biomed Central

C 2012 Khan et al.; licensee BioMed Central Ltd. This is an open access article distributed under the terms of the Creative Commons Attribution License (http://creativecommons.org/licenses/by/2.0), which permits unrestricted use, distribution, and reproduction in any medium, provided the original work is properly cited. 
multiple binding sites. Moonlighting proteins have been found to be involved in molecular functions ranging from diseases and disorders $[16,40,41]$ to immune systems $[40,41]$.

In this work, we have analyzed the ability of existing function prediction methods to correctly identify diverse functions of experimentally identified moonlighting proteins [42]. We have collected Gene Ontology (GO) term annotations of these proteins from the UniProt database and manually classified these annotations into two distinct functions. Based on the GO annotations, we have examined the prediction performance of PSI-BLAST and two other major sequence based function prediction methods, the Protein Function Prediction (PFP) and the Extended Similarity Group (ESG) method.

Overall, PFP showed higher average recall than PSIBLAST and ESG. ESG showed lower recall as compared with PFP and PSI-BLAST, although it has a higher precision. The results suggest that the functional diversity of the moonlighting proteins can be captured if weakly similar sequences are considered among a broad range of similar sequence sets.

\section{Methods}

\section{Function prediction methods}

In this section we briefly describe the three AFP methods we examined, PFP, ESG, and PSI-BLAST. Since PFP $[8,9]$ and ESG [10] have been published in earlier works, please refer to the original works for more details.

\section{Protein function prediction (PFP) algorithm}

The PFP algorithm uses PSI-BLAST to obtain sequence hits for a target sequence and predict $\mathrm{GO}$ function annotations. PFP computes the score to GO term $f_{a}$ as follows:

$$
s(f a)=\sum_{i=1}^{N} \sum_{j=1}^{N f u n c(i)}((-\log (\text { E_value }(i))+b) P(f a \mid f j)),
$$

where $N$ is the number of sequence hits considered in the PSI-BLAST hits up to E-value of 100, $N f u n c(i)$ is the number of $\mathrm{GO}$ annotations for the sequence hit $i$, $E_{-}$value $(i)$ is the PSI-BLAST E_value for the sequence hit $i, f_{j}$ is the $j$-th annotation of the sequence hit $i$, and constant $b$ takes value $2\left(=\log _{10} 100\right)$ to keep the score positive as retrieved sequences up to $E_{-}$value of 100 are used $\left(-\log \left(\mathrm{E}_{-}\right.\right.$value(i) $)+\mathrm{b}=-\log _{10}(100)+2=0$, when E_value $=100)$. The conditional probabilities $P\left(f_{a} \mid f_{j}\right)$ is to consider co-occurrence of GO terms in single sequence annotation, which is computed as the ratio of number of proteins co-annotated with GO terms $f_{a}$ and $f_{j}$ as compared with genes annotated with the term $f_{j}$. To take into account the hierarchical structure of the GO, PFP transfers the raw score to the parental terms by computing the proportion of proteins annotated with $f_{a}$ relative to all proteins that belong to the parental GO term in the database. The score of a GO term computed as the sum of the directly computed score by Eqn. 1 and the ones from the parental propagation is called the raw score.

\section{Extended Similarity Group (ESG) algorithm}

ESG recursively performs PSI-BLAST searches from sequence hits obtained in the initial search from the target sequence, thereby performing multi-level exploration of the sequence similarity space around the target protein. Each sequence hit in a search is assigned a weight that is computed as the proportion of the $-\log \left(E_{-}\right.$value) of the sequence relative to the sum of $-\log \left(E_{-}\right.$value $)$ from all the sequence hits considered in the search of the same level. This weight is assigned for GO terms annotating the sequence hit. The weights for GO terms found in the second level search are computed in the same fashion. Ultimately the score for a GO term is computed as the total weight from the two levels of the searches. The score for each GO term ranges from 0 to 1.0 .

\section{PSI-BLAST}

PSI-BLAST search is performed with a default setting with maximum of three iterations. Then the top hits with an E_value score better than 0.01 that have annotations is used for transferring annotation to the query sequence. The BLAST predictions were ranked according to $-\log \left(E_{-}\right.$value $)+2$ for each of the prediction. In addition to the default BLOSUM62, which is the default amino acid similarity matrix, we also tested PSI-BLAST performance using BLOSUM45 and BLOSUM30.

\section{Results}

We analyzed the performances of PFP, ESG, and PSIBLAST in predicting the functional diversity of 19 moonlighting proteins. The 19 moonlighting proteins were taken from a review article [42]. These proteins have two diverse and distinct functions. According to the verbal description of the two diverse functions of the proteins, we classified GO terms of these proteins in UniProt into four classes: Terms that belong to the major moonlighting function of the protein (Function 1 ); those which belong to the second moonlighting function (Function 2); terms which belong to both functions; and terms that do not belong to either of the functions. The list of the moonlighting proteins and their classified GO terms are made available at http://kiharalab.org/ MoonlightingProtein_Dataset1/.

The raw score of PFP predictions has a large range of values. Up to $1000 \mathrm{GO}$ term predictions were sorted by their raw score and plotted at an interval of 10. ESG 
predictions have a score range of 0 to 1.0 , and 100 cutoffs are used within this range. PSI-BLAST predictions are ranked by $-\log \left(\mathrm{E}_{-}\right.$value $)+2$, and 100 score cutoffs are used from 4 (E_value of 0.01 ) to 45 (E_value of $10^{-43}$ ). To compare the prediction performances of the methods, we computed precision and recall. Precision is defined as $\mathrm{TP} /(\mathrm{TP}+\mathrm{FP})$ and recall is defined as $\mathrm{TP} /(\mathrm{TP}+\mathrm{FN})$, where TP and FP denote true and false positive, respectively, and FN denote false negative. All predictions by the three methods are propagated to the root of the GO hierarchy, so are the true annotations for the proteins.

\section{Average Precision-Recall performance of PFP, ESG, and PSI-BLAST}

In Figure 1, the average precision and recall of PFP, ESG, and PSI-BLAST for all the GO terms of the 19 moonlighting proteins are shown. It is shown that ESG perform significantly better than the other two methods in the recall range of $0.4-0.7$. ESG has better precision than BLAST within recall range of $0.37-0.66$. PFP predictions ranked with raw score (Eq. 1 in Methods) reaches the highest recall. In Figure 2 we show the performance of the methods in terms of recall values at 100 cutoff scores (with all the GO annotations of the proteins considered). It is apparent from this plot that PFP showed higher recall than PSI-BLAST, and ESG. ESG has lowest recall within the cutoff range of 0.090.88 .

In Figure 2B, the performance was evaluated where only the GO annotations for the two moonlighting functions (Function 1 and Function 2) are taken into account as the target annotations. The prediction performance for the moonlighting functions is essentially the same as those measured for the all GO term annotations (Figure 2A).

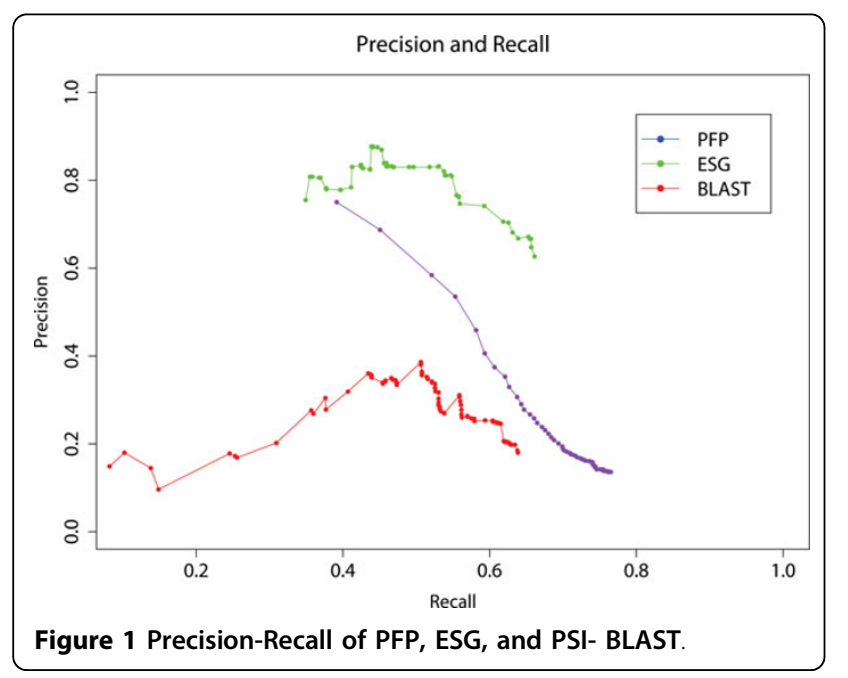

\section{Recall at individual proteins}

Next In Figure 3, we plotted the recall for the three methods for each of the 19 moonlighting proteins separately. The cutoff of the prediction scores used are 0.5 for PFP, 0.35 for ESG, and E_value 0.01 for PSI-BLAST. The PFP cutoff of 0.5 will yield the maximum of $500 \mathrm{GO}$ term predictions. The score cutoff value of 0.35 for ESG is an optimal cutoff score established in the previous work [10]. E_value 0.01 for PSI-BLAST is a standard cutoff used in general for homology search. In addition to default PSIBLAST setting with BLOSUM62, we have also added the predictions of two more versions of PSI-BLAST, with BLOSUM45 and BLOSUM30 scoring matrices (BL+bls45 and $\mathrm{BL}+30$ in Figure 3, respectively) to consider more divergent sequences in the homology search.

When all the GO terms are considered (Figure 3A), PFP showed higher recall than PSI-BLAST for almost all the cases (except for proteins 2 and 4, which are ties). ESG has similar recall of predictions as PSI-BLAST for proteins 14 and 17, slightly higher recall for proteins 6 , 12 and 15 than PSI-BLAST (BLOSUM62), and a lower recall than PFP and PSI-BLAST for the rest of the proteins. Recall by PSI-BLAST improved when BLOSUM45 was used. In the head-to-head comparison against PFP, PSI-BLAST with BLOSUM45 showed a higher recall than PFP for eight proteins while PFP had a higher recall in ten cases (there was a tie). PSI-BLAST with BLOSUM30 failed to predict any GO terms above E_value of 0.01 for twelve proteins (Figure 3A). Overall, PFP and PSI-BLAST with BLOSUM45 showed higher recall than the rest of the methods. We see a similar performance pattern for the five methods when we consider only the GO terms belonging to moonlighting function 1 and function 2 of the proteins (Figure 3B). Again PSI-BLAST with BLOSUM45 showed comparable performance to PFP. PSI-BLAST with BLOSUM45 had a higher recall than PFP in seven cases while PFP was higher in ten cases (again there was a tie).

These results indicate that the PFP can find moonlighting GO terms that are missed by regular PSI-BLAST searches for quite a lot of cases. The strength of PFP is its coverage of a large number of sequences, by including weakly similar sequences into consideration for annotation transfer. On the other hand, ESG puts more weight on the consensus sequences that have strong similarity with the query protein among all the sequences that it encounters along multiple iterations. Thus, although ESG provides a higher precision on the predictions among all three methods (Figure 1), it fails to detect the functional variations in a number of cases. These results suggest that the functional diversity of the moonlighting proteins could be captured by taking weakly similar sequences into consideration among a broad range of similar sequences. 

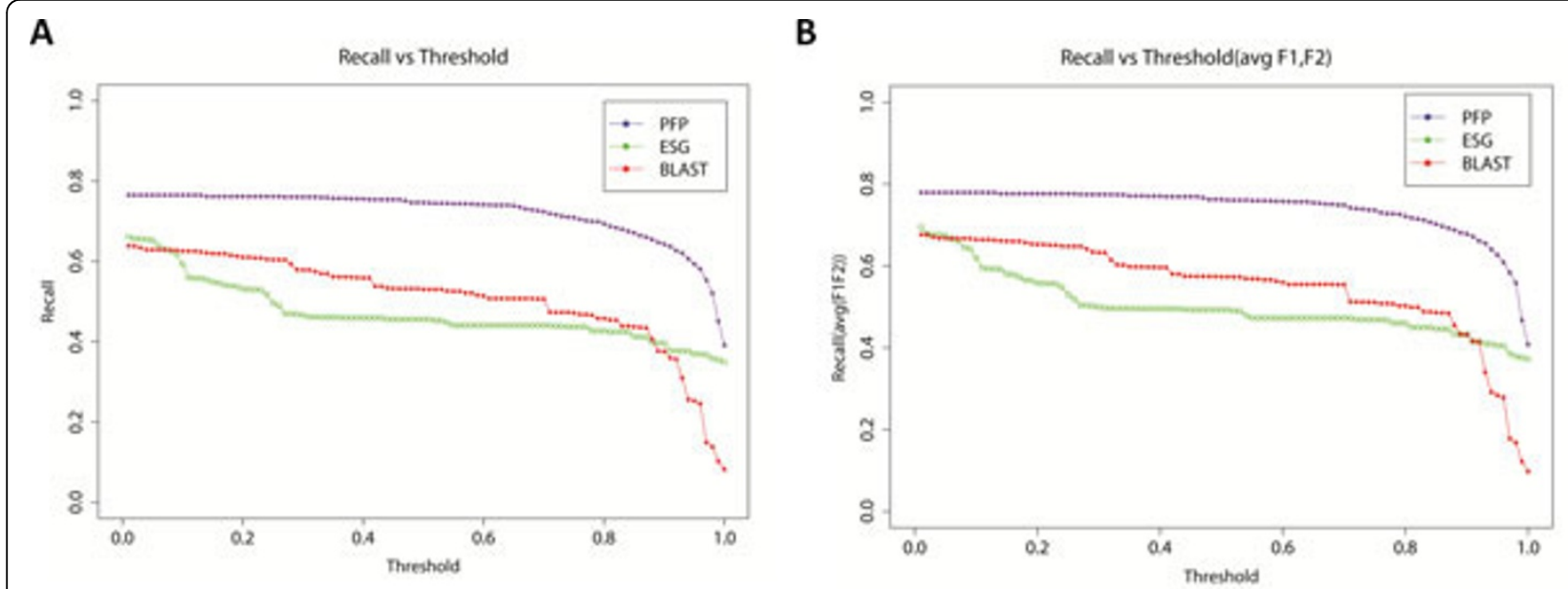

Figure 2 Recall of PFP, ESG and PSI-BLAST at each threshold. A, Recall where all the GO annotations for proteins are considered. B, Recall where only the GO annotations labeled as Function 1 or Function 2 for proteins are considered.

A

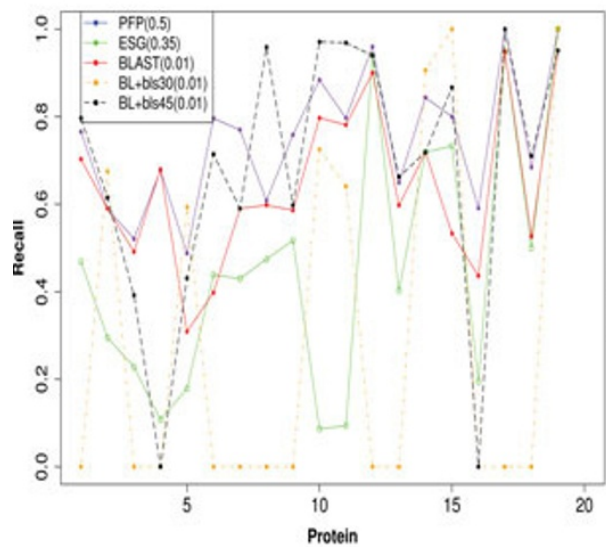

B

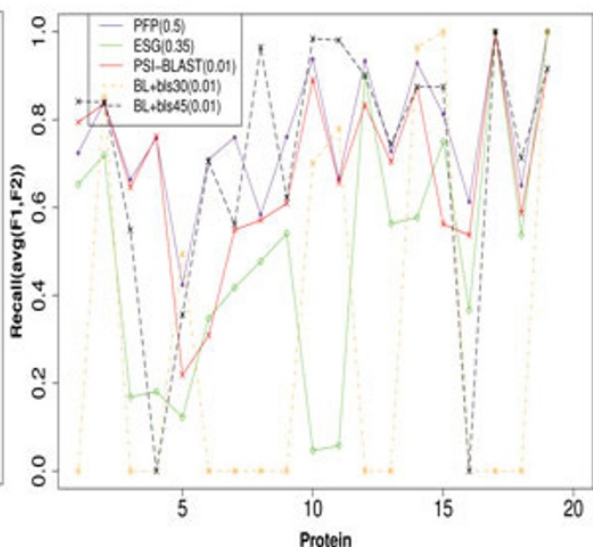

Figure 3 Recall of PFP, ESG, PSI-BLAST, PSI-BLAST with BLOSUM62 (default), BLOSUM30, and BLOSUM45 scoring matrix for each protein. Score thresholds used for the methods are PFP: 0.5, ESG: 0.35 and PSI-BLAST: 0.01 A, Recall where all the GO annotations for proteins are considered. B, Recall where only the GO annotations labeled as Function 1 or Function 2 for proteins are considered.

\section{Conclusion}

The identification of moonlighting functions of a protein is important for automatic function predictions. We have analyzed the performances of PFP, ESG, and PSIBLAST in predicting the functional diversity of moonlighting proteins. PFP shows overall better performance in predicting diverse moonlighting functions as compared with PSI-BLAST and ESG. Recall by PSI-BLAST greatly improved when BLOSUM45 was used instead of BLOSUM62. This analysis indicates that considering weakly similar sequences in prediction enhances the performance of sequence based AFP methods in predicting functional diversity of moonlighting proteins.

\section{Acknowledgements}

This work was supported by the National Institute of General Medical Sciences of the National Institutes of Health (R01GM075004, R01GM097528) and the National Science Foundation (EF0850009, IIS0915801, IOS1127027) and National Research Foundation of Korea Grant (NRF-2011-220-C00004). MC is supported by Bilsland Dissertation fellowship at Purdue University. This article has been published as part of BMC Proceedings Volume 6 Supplement 7, 2012: Proceedings from the Great Lakes Bioinformatics Conference 2012. The full contents of the supplement are available online at http://www.biomedcentral.com/bmcproc/supplements/6/S7.

\section{Author details}

${ }^{1}$ Department of Computer Science, Purdue University, 305 N. University Street, West Lafayette, Indiana 47907, USA. 'Department of Biological Sciences, Purdue University, 915 W. State Street, West Lafayette, Indiana 47907, USA. ${ }^{3}$ EA3900-BIOPI Biologie des Plantes et Innovation, Université de Picardie Jules Verne, 33 Rue St Leu, 80039 Amiens, France. 


\section{Authors' contributions}

IKK did the experiment and drafted the manuscript. MC has developed ESC and helped in doing the experiment. CR has participated in classifying $\mathrm{GO}$ terms of the moonlighting proteins. DK conceived the study and participated in its design and coordination, as well as drafting and finalizing the manuscript. All authors read and approved the final manuscript.

\section{Competing interests}

The authors declare that they have no competing interests.

Published: 13 November 2012

\section{References}

1. Hawkins T, Kihara D: Function prediction of uncharacterized proteins. Journal of bioinformatics and computational biology 2007, 5:1-30.

2. Hawkins T, Chitale M, Kihara D: New paradigm in protein function prediction for large scale omics analysis. Mol BioSyst 2008, 4:223-231.

3. Altschul SF, Gish W, Miller W, Myers EW, Lipman DJ: Basic local alignment search tool. Journal of molecular biology 1990, 215:403-410.

4. Pearson WR: Rapid and sensitive sequence comparison with FASTP and FASTA. Methods in enzymology 1990, 183:63-98.

5. Bru C, Courcelle E, Carrere S, Beausse Y, Dalmar S, Kahn D: The ProDom database of protein domain families: more emphasis on 3D. Nucleic acids research 2005, 33:D212-D215.

6. Finn RD, Mistry J, Schuster-Bockler B, Griffiths-Jones S, Hollich V, Lassmann T, et al: Pfam: clans, web tools and services. Nucleic acids research 2006, 34:D247-D251.

7. Hunter S, Apweiler R, Attwood TK, Bairoch A, Bateman A, Binns D, et al: InterPro: the integrative protein signature database. Nucleic acids research 2009, 37:D211-D215.

8. Hawkins T, Luban S, Kihara D: Enhanced automated function prediction using distantly related sequences and contextual association by PFP. Protein Science 2006, 15:1550-1556.

9. Hawkins T, Chitale M, Luban S, Kihara D: PFP: automated prediction of gene ontology functional annotations with confidence scores using protein sequence data. Proteins: Structure, Function, and Bioinformatics 2009, 74:566-582.

10. Chitale M, Hawkins T, Park C, Kihara D: ESG: extended similarity group method for automated protein function prediction. Bioinformatics 2009, 25:1739-1745

11. Martin D, Berriman M, Barton G: GOtcha: a new method for prediction of protein function assessed by the annotation of seven genomes. BMC Bioinformatics 2004, 5:178-194.

12. Khan S, Situ G, Decker K, Schmidt CJ: GoFigure: automated Gene Ontology annotation. Bioinformatics 2003, 19:2484-2485.

13. Wass MN, Sternberg MJ: ConFunc-functional annotation in the twilight zone. Bioinformatics 2008, 24:798-806.

14. Engelhardt $\mathrm{BE}$, Jordan MI, Muratore KE, Brenner SE: Protein molecular function prediction by Bayesian phylogenomics. PLoS Comput Biol 2005, 1:e45.

15. Krishnamurthy N, Brown D, Sj+|lander K: FlowerPower: clustering proteins into domain architecture classes for phylogenomic inference of protein function. BMC Evolutionary Biology 2007, 7:512.

16. Storm CEV, Sonnhammer ELL: Automated ortholog inference from phylogenetic trees and calculation of orthology reliability. Bioinformatics 2002, 18:92.

17. Brown MPS, Grundy WN, Lin D, Cristianini N, Sugnet CW, Furey TS, et al: Knowledge-based analysis of microarray gene expression data by using support vector machines. Proceedings of the National Academy of Sciences 2000, 97:262.

18. Eisen MB, Spellman PT, Brown PO, Botstein D: Cluster analysis and display of genome-wide expression patterns. Proceedings of the National Academy of Sciences 1998, 95:14863.

19. Gao L, Li X, Guo Z, Zhu M, Li Y, Rao S: Widely predicting specific protein functions based on protein-protein interaction data and gene expression profile. Sci China C Life Sci 2007, 50:125-134.

20. Khatri P, Dr-âghici S: Ontological analysis of gene expression data: current tools, limitations, and open problems. Bioinformatics 2005, 21:3587-3595.

21. van Noort V, Snel B, Huynen MA: Predicting gene function by conserved co-expression. TRENDS in Genetics 2003, 19:238-242.
22. Gherardini PF, Helmer-Citterich M: Structure-based function prediction: approaches and applications. Briefings in functional genomics \& proteomics 2008, 7:291-302.

23. Marti-Renom M, Rossi A, Al-Shahrour F, Davis F, Pieper U, Dopazo J, et al: The AnnoLite and AnnoLyze programs for comparative annotation of protein structures. BMC Bioinformatics 2007, 8:54.

24. Martin $A C R$, Orengo $C A$, Hutchinson $E G$, Jones $S$, Karmirantzou $M$, Laskowski RA, et al: Protein folds and functions. Structure 1998, 6:875-884.

25. Pal $D$, Eisenberg D: Inference of protein function from protein structure. Structure 2005, 13:121-130.

26. Ponomarenko JV, Bourne PE, Shindyalov IN: Assigning new GO annotations to protein data bank sequences by combining structure and sequence homology. Proteins: Structure, Function, and Bioinformatics 2005, 58:855-865.

27. Thornton JM, Todd AE, Milburn D, Borkakoti N, Orengo CA: From structure to function: approaches and limitations. nature structural biology 2000, 7:991-994

28. Chikhi R, Sael L, Kihara D: Real-time ligand binding pocket database search using local surface descriptors. Proteins: Structure, Function, and Bioinformatics 2010, 78:2007-2028.

29. Sael L, Kihara D: Binding ligand prediction for proteins using partial matching of local surface patches. International Journal of Molecular Sciences 2010, 11:5009-5026.

30. Sael L, Chitale M, Kihara D: Structure- and sequence-based function prediction for non-homologous proteins. Journal of Structural and Functional Genomics. Journal of Structural and Functional Genomics 2012, Ref Type: In Press.

31. Brun C, Chevenet F, Martin D, Wojcik J, Guenoche A, Jacq B: Functional classification of proteins for the prediction of cellular function from a protein-protein interaction network. Genome Biol 2003, 5:R6.1-R6.13.

32. Chua HN, Sung WK, Wong L: Exploiting indirect neighbours and topological weight to predict protein function from protein-protein interactions. Bioinformatics 2006, 22:1623-1630.

33. Letovsky S, Kasif S: Predicting protein function from protein/protein interaction data: a probabilistic approach. Bioinformatics 2003, 19(Suppl 1):i197-i204.

34. Nariai N, Kolaczyk ED, Kasif S: Probabilistic protein function prediction from heterogeneous genome-wide data. PLoS One 2007, 2:e337.1-e337.7.

35. Sharan R, Ulitsky I, Shamir R: Network-based prediction of protein function. Mol Syst Biol 2007, 3:88-100.

36. Deng $M, T u Z$, Sun $F$, Chen T: Mapping gene ontology to proteins based on protein-protein interaction data. Bioinformatics 2004, 20:895-902.

37. Jeffery CJ: Moonlighting Proteins. Trends in Biochemical Sciences 1999, 24:8-11.

38. Jeffery CJ: Moonlighting Proteins: old proteins learning new tricks. TRENDS in Genetics 2003, 19:415-417.

39. Gancedo C, Flores CL: Moonlighting proteins in yeasts. Microbiology and Molecular Biology Reviews 2008, 72:197-210.

40. Jeffery CJ: Proteins with neomorphic moonlighting functions in disease. IUBMB Life 2011, 63:489-494.

41. Ovadi J: Moonlighting Proteins in Neurological Disorders. IUBMB Life 2011, 63:453-456.

42. Huberts DHEW, Klei IJvd: Moonlighting proteins: an intriguing mode of multitasking. Biochim Biophys Acta 2010, 1803:520-525.

doi:10.1186/1753-6561-6-S7-S5

Cite this article as: Khan et al: Evaluation of function predictions by PFP, ESG, and PSI-BLAST for moonlighting proteins. BMC Proceedings 2012 6(Suppl 7):S5 